University of New Hampshire

University of New Hampshire Scholars' Repository

Space Science Center

Institute for the Study of Earth, Oceans, and

Space (EOS)

1998

\title{
A hard X-ray solar flare polarimeter design based on scintillating fibers
}

James M. Ryan

University of New Hampshire, James.Ryan@unh.edu

Mark L. McConnell

University of New Hampshire - Main Campus, mark.mcconnell@unh.edu

D J. Forrest

University of New Hampshire - Main Campus

John R. Macri

University of New Hampshire - Main Campus, John.Macri@unh.edu

M McClish

University of New Hampshire - Main Campus

See next page for additional authors

Follow this and additional works at: https://scholars.unh.edu/ssc

Part of the Astrophysics and Astronomy Commons

\section{Recommended Citation}

A hard X-ray solar flare polarimeter design based on scintillating fibers Ryan, J. M. and McConnell, M. L. and Forrest, D. J. and Macri, J. and McClish, M. and Vestrand, W. T., AIP Conference Proceedings, 450, 586-591 (1998), DOI:http://dx.doi.org/10.1063/1.56962

This Conference Proceeding is brought to you for free and open access by the Institute for the Study of Earth, Oceans, and Space (EOS) at University of New Hampshire Scholars' Repository. It has been accepted for inclusion in Space Science Center by an authorized administrator of University of New Hampshire Scholars' Repository. For more information, please contact Scholarly.Communication@unh.edu. 


\section{Authors}

James M. Ryan, Mark L. McConnell, D J. Forrest, John R. Macri, M McClish, and W T. Vestrand 


\section{AIP $\mid$ proceedings}

\section{A hard X-ray solar flare polarimeter design based on scintillating fibers}

J. M. Ryan, M. L. McConnell, D. J. Forrest, J. Macri, M. McClish, and W. T.

Vestrand

Citation: AIP Conference Proceedings 450, 586 (1998); doi: 10.1063/1.56962

View online: http://dx.doi.org/10.1063/1.56962

View Table of Contents:

http://scitation.aip.org/content/aip/proceeding/aipcp/450?ver=pdfcov

Published by the AIP Publishing

Articles you may be interested in

Lanthanum Chloride Scintillator for Xray Detection

AIP Conf. Proc. 879, 1156 (2007); 10.1063/1.2436269

Geometrical interpretation of the hard Xray flux asymmetry in the flare's footpoints AIP Conf. Proc. 801, 403 (2005); 10.1063/1.2141914

High-resolution x-ray crystal spectrometer/polarimeter at torus experiment for technology oriented research-94

Rev. Sci. Instrum. 72, 2566 (2001); 10.1063/1.1370558

Hard x-ray spectrometers for NIF (abstract)

Rev. Sci. Instrum. 72, 1201 (2001); 10.1063/1.1326019

Development of a hard x-ray polarimeter for gamma-ray bursts AIP Conf. Proc. 428, 889 (1998); 10.1063/1.55415 


\title{
A Hard X-Ray \\ Solar Flare Polarimeter Design Based on Scintillating Fibers
}

\author{
J.M. Ryan, M.L. McConnell, D.J. Forrest, \\ J. Macri, M. McClish and W.T. Vestrand
}

Space Science Center, University of New Hampshire, Durham, NH 03824

\begin{abstract}
We have developed a design for a Compton scatter polarimeter to measure the polarization of hard X-rays (50-300 keV) from solar flares. The modular design is based on an annular array of scintillating fibers coupled to a 5-inch position-sensitive PMT. Incident photons scatter from the fiber array into a small array of $\mathrm{NaI}$ detectors located at the center of the annulus. The location of the interactions in both the fiber array and in the $\mathrm{NaI}$ array can be used to measure the linear polarization of the incident flux. This compact design may be well-suited to a variety of astrophysical applications. An extensive series of Monte Carlo simulations has been performed to characterize this design.
\end{abstract}

\section{INTRODUCTION}

The measurement of hard X-ray polarization in solar flares would provide insights into the geometry of the electron acceleration process. In particular, such polarization measurements would indicate the extent to which the electrons are beamed. Here we report on the development of a hard X-ray polarimeter for solar flares that is based on the use of scintillating fibers. Due to its relatively large FoV, this design may also be useful in studies of $\gamma$-ray bursts.

The basic physical process used to measure linear polarization of hard $\mathrm{X}$ rays $(100-300 \mathrm{keV})$ is Compton scattering. The measurement is based on the fact that the incident photons tend to be scattered at right angles to the incident electric field vector. A Compton scatter polarimeter consists of two detectors that are used to determine the energies of both the scattered photon and the scattered electron. One detector (the scattering detector) provides the medium for the Compton interaction to take place. This detector must be designed to maximize the probability of a single Compton interaction with a subsequent escape of the scattered photon. The primary purpose of the 
second detector (the calorimeter) is to absorb the full energy of the scattered photon. To be recorded as a polarimeter event, an incident photon Compton scatters from one (and only one) of the scattering detectors into the central calorimeter. The incident photon energy can be determined from the sum of the energy losses in both detectors and the scattering angle can be determined by the azimuthal angle of the associated scattering detector. When the polarimeter is arranged so that the incident flux is parallel to the symmetry axis, unpolarized radiation will produce an axially symmetric coincidence rate. If the incident radiation is linearly polarized, then the coincidence rate will show an azimuthal asymmetry whose phase depends on the position angle of the incident radiation's electric vector and whose magnitude depends on the degree of polarization.

\section{LABORATORY PROTOTYPE}

In an earlier paper, we discussed a polarimeter design consisting of a ring of twelve individual scattering detectors (composed of low- $Z$ plastic scintillator) surrounding a single $\mathrm{NaI}$ calorimeter [1]. The characteristics of this design were investigated using a series of Monte Carlo simulations (based on a modified version of GEANT). We have recently prototyped this design in the laboratory to validate our Monte Carlo code. For prototype testing, we set up a semicircular array around a central $\mathrm{NaI}$ detector, eliminating the redundancy and simplifying the hardware and associated electronics. Seven plastic scintillators (each $5.5 \mathrm{~cm} \times 5.5 \mathrm{~cm} \times 7.0 \mathrm{~cm}$ in size) were positioned at a radius of $15 \mathrm{~cm}$ from a $7.6 \mathrm{~cm}$ diameter $\times 7.6 \mathrm{~cm}$ high cylindrical $\mathrm{NaI}(\mathrm{Tl})$ detector.

Polarized photons were generated by Compton scattering photons from a radioactive source [2]. The exact level of polarization is dependent on both the initial photon energy and the photon scatter angle. The use of plastic scintillators as a scattering block permits the electronic tagging of the scattered (polarized) photons. This is used to provide a coincidence signal to the polarimeter. For our laboratory measurements we used a ${ }^{137} \mathrm{Cs}$ source to generate a beam of polarized $288 \mathrm{keV}$ photons.

The laboratory data (Figure 1) led to a measured polarization value of $64.0 \%( \pm 3.0 \%)$, in good agreement with the estimated value of $50-60 \%$ based on analytical estimates [3]. This result demonstrates: a) the ability of a simple Compton scatter polarimeter to measure hard X-ray polarization; $b$ ) the ability of our Monte Carlo code to predict the polarimeter response; and c) the ability to generate a source of polarized photons using a simple scattering technique. In another laboratory measurement (Figure 2), the plane of polarization of the incident beam was rotated $\sim 45^{\circ}$ with respect to that used in the first set of data. The measured shift of $50.4^{\circ}$ in the polarization vector is consistent with the uncertainties in our experimental setup. 


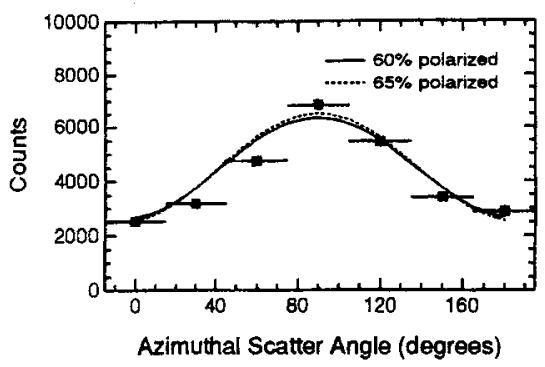

FIGURE 1. The prototype response to an on-axis polarized beam. The smooth curves represent simulation results.

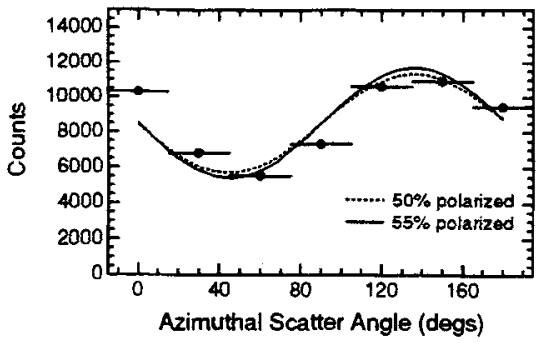

FIGURE 2. The prototype response with the polarization vector rotated $45^{\circ}$ with respect to that in Figure 1.

\section{A NEW DESIGN CONCEPT}

There are at least two possible means of improving the polarimeter performance: 1) by more precisely measuring the scattering geometry of each event; and 2) by rejecting those events that undergo multiple Compton scattering within the scattering elements. (Our simulations indicate that roughly $30-40 \%$ of the events recorded in the prototype polarimeter as valid events involved multiple scattering within a single scatter element.) Improvements in either area will lead directly to a more clearly defined modulation and, therefore, a better polarization sensitivity.

We have developed a new design that places an entire device on the front end of a single 5-inch diameter position-sensitive PMT (PSPMT) [4]. A bundle of scintillation fibers (each with a cross section of $4 \mathrm{~mm} \times 4 \mathrm{~mm}$ ) provides the improved spatial resolution in the scattering elements. The bundle is in the form of an annulus with an outside diameter of $10 \mathrm{~cm}$ and an inside diameter of $4 \mathrm{~cm}$. A $2 \times 2$ array of $1 \mathrm{~cm}$ inorganic scintillators is positioned within the annulus, each scintillator being coupled to its own independent PMT for light collection and signal timing. Figure 3 shows a schematic view of such an assembly.

Monte Carlo simulations have been used to determine the characteristics of this design. Figure 4 shows the modulation curves that result from completely polarized incident radiation at two different energies ( $100 \mathrm{keV}$ and $300 \mathrm{keV})$. Figures 5 and 6 show the modulation factor and the effective area, respectively, as a function of energy. The low energy response is very sensitive to the energy threshold in the fiber array. Figure 7 shows the off-axis response of the design, which suggests a useful FoV of at least one steradian.

We are currently involved in a series of laboratory tests designed to evaluate the characteristics of a PSPMT / fiber bundle detector system. These tests will help determine the achievable energy resolution and, more importantly, the achievable energy threshold of the fiber array. The precise energy threshold level of the fiber bundle will have a major impact on the final characteristics of 


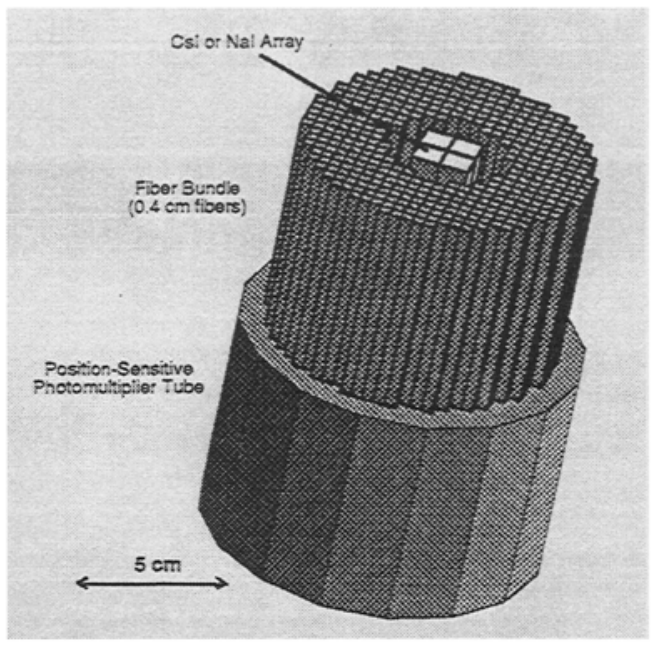

FIGURE 3. Schematic diagram of a polarimeter module.

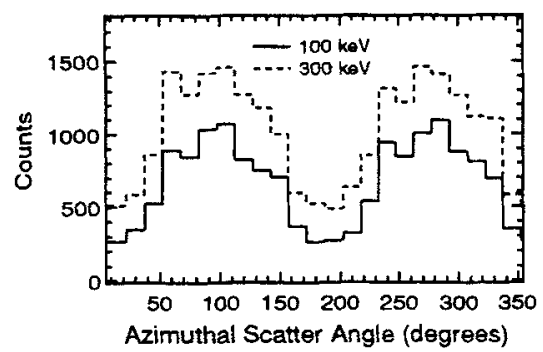

FIGURE 4. Simulated modulation curves (counts versus azimuthal scatter angle) at energies of 100 and $300 \mathrm{keV}$.

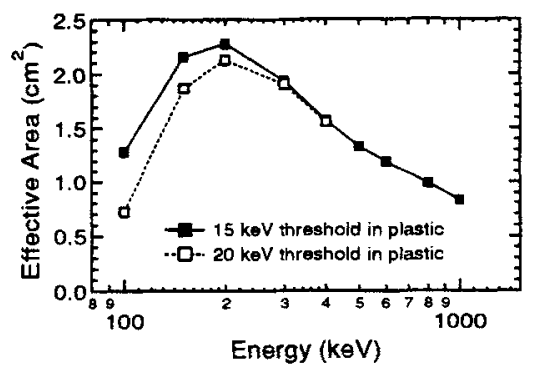

FIGURE 5. Effective area versus energy.

the polarimeter module. In particular, the fiber bundle threshold will dictate the low energy threshold of the polarimeter module and also the characteristics of the polarimeter module (in terms of effective area and modulation factor) at low energies. Our goal is to achieve an energy threshold in the range of $10-30 \mathrm{keV}$ for the fiber bundle.

\section{SUMMARY}

We anticipate that this design would be used in the context of a (not necessarily contiguous) array of polarimeter modules. In the case of solar flares, we calculate that an array of 4 modules would be capable of measuring polarization levels down to a few percent in X-class flares. A larger array of 16 


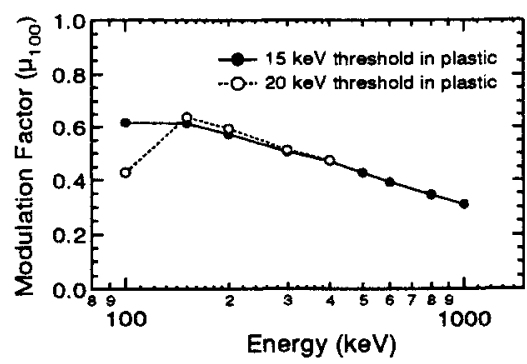

FIGURE 6. Modulation factor versus en- FIGURE 7. The modulation factor and efergy.

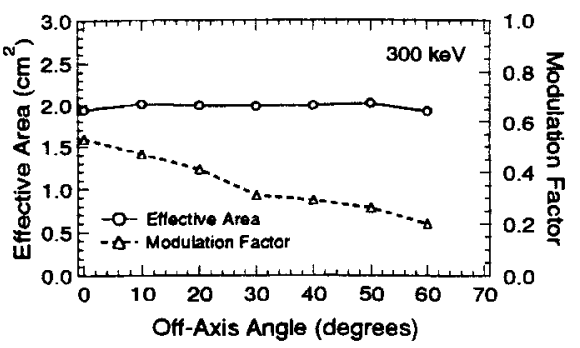

fective area as a function of incidence angle for a photon energy of $300 \mathrm{keV}$.

modules would be capable of measuring solar flare polarization levels below $1 \%$ for the largest events and would also be capable of measuring polarization levels down to about $15 \%$ in some of the largest $\gamma$-ray bursts [12]

The use of polarimetry in X-ray and $\gamma$-ray astronomy has so far been largely limited to energies below $100 \mathrm{keV}[5-7,2,8]$, with an emphasis on the study of non-transient sources. Several higher energy experiments offer polarimetry as a secondary capability $[9,10]$. Although designs similar to that proposed here have been discussed in the literature $[6,11]$, we are unaware of any other active effort to specifically measure polarization in solar flares and $\gamma$-ray bursts at energies above $100 \mathrm{keV}$.

\section{ACKOWLEDGEMENT}

This work has been supported by NASA grant NAGW-5704.

\section{REFERENCES}

1. M. McConnell, D. Forrest, K. Levenson, and W.T. Vestrand, "The design of a gamma-ray burst polarimeter", in AIP Conf. Proc. 280, Compton Gamma-Ray Observatory, M. Friedlander, N. Gehrels and D.J. Macomb, Eds. New York: AIP, 1993, pp. 1142-1146.

2. H. Sakurai, M. Noma, and H. Niizeki, "A hard X-ray polarimeter utilizing Compton scattering", in SPIE Conf. Proc., vol. 1343, pp.512-518, 1990.

3. W.H. McMaster, "Matrix representation of polarization", Reviews of Mod. Phys., vol. 33, no. 1, pp. 8-28, January 1961.

4. M.L. McConnell, et al., "Development of a hard X-ray polarimeter for solar flares and gamma-ray bursts", submitted to IEEE Trans. Nucl. Sci., 1998.

5. R. Novick, "Stellar and solar X-ray polarimetry", Space Science Reviews, vol. 18 , pp. 389-408, 1975. 
6. G. Chanan, A.G. Emslie, and R. Novick, "Prospects for solar flare X-ray polarimetry", Solar Physics, vol. 118, pp. 309-319, 1988.

7. P. Kaaret, et al., "The Stellar X-ray Polarimeter - a focal plane polarimeter for the Spectrum X-Gamma mission", Optical Engineering, vol. 29, pp. 773-780, July 1990 .

8. E. Costa, M.N. Cinti, M. Feroci, G. Matt, and M. Rapisarda, "Scattering polarimetry for X-ray astronomy by means of scintillating fibers", SPIE Conf. Proc, vol. 2010, pp. 45-56, 1993.

9. E. Aprile, A. Bolotnikov, D. Chen, R. Mukherjee and F. Xu, "The polarization sensitivity of the liquid zenon imaging telescope", ApJ Supp, vol. 92, pp. 689692, June 1994.

10. T.J. O'Neill, et al., "Tracking, imaging and polarimeter properties of the TIGRE instrument", Astron. Astrophys. Suppl. Ser., vol., 120, pp. C661-C664.

11. T.L. Cline, et al., "A Gamma-Ray Burst Polarimeter Study", in Proceedings of the 25th Internat. Cosmic Ray Conf., vol. 5, pp. 25-28, 1997.

12. M.L. McConnell, et al., "Development of a hard X-ray polarimeter for gammaray bursts", to be published in AIP Conf. Proc., 4th Gamma Ray Bursts Symposium, 1998. 